\title{
Replicating Meyerhoff for Inclusive Excellence in STEM
}

\author{
By Mariano R. Sto. Domingo ${ }^{1}$, Starlette Sharp ${ }^{2,3}$, Amy Freeman², Thomas Freeman, Jr. ${ }^{4}$, Keith Harmon ${ }^{5}$, Mitsue Wiggs ${ }^{5}$, Viji Sathy ${ }^{6}$, Abigail T. Panter ${ }^{6,7}$, Leticia \\ Oseguera $^{8}$, Shuyan Sun ${ }^{1}$, Mary Elizabeth Williams ${ }^{9}$, Joseph Templeton ${ }^{10,11}$, Carol L. Folt ${ }^{10,12}$, Eric J. Barron ${ }^{13}$, Freeman A. Hrabowski III ${ }^{14}$, Kenneth I. Maton ${ }^{1}$, \\ Michael Crimmins ${ }^{11}$, Charles R. Fisher ${ }^{9}$, and Michael F. Summers ${ }^{15}$,*
}

Ethnic minorities comprise rapidly growing portions of the populations of most developed countries (1) but are underrepresented in fields of science, technology, engineering, and mathematics (STEM) $(2,3)$. Efforts to increase diversity in the STEM workforce, important for developing more effective approaches to group problem solving (4-6), have been underway in the U.S. for decades, but widespread impact remains relatively low (3). The Meyerhoff Scholars Program (MYS) at the University of Maryland Baltimore County (UMBC) provides a promising model for increasing retention and academic performance of URM undergraduates in STEM and for preparing those undergraduates to pursue and succeed in graduate and professional programs $(7,8)$. Although MYS is nearly 30 years old and outcomes for African American STEM majors have been extensively documented (see $(7,8)$, and references there-in) no other majority university (not meeting the definition of being a minority serving institution (MSI) (9)) has achieved similar outcomes (10). We describe here some promising early indicators that an inter-institutional partnership approach can help enable MYS-like outcomes at majority universities with different URM compositions, geographies, and institutional sizes and cultures: The University of North Carolina at Chapel Hill (UNC) and Pennsylvania State University at University Park (PSU).

MYS includes students of all ethnicities

\footnotetext{
${ }^{1}$ Department of Psychology, ${ }^{5}$ Meyerhoff Scholars Program, ${ }^{14}$ Office of the President, ${ }^{15}$ Department of Chemistry and Biochemistry and Howard Hughes Medical Institute, University of Maryland Baltimore County (UMBC), 1000 Hilltop Circle, Baltimore, MD 21250, USA. ${ }^{2}$ Millennium Scholars Program, ${ }^{3}$ Department of Curriculum and Instruction, ${ }^{8}$ Department of Education Policy Studies, ${ }^{9}$ Office of the Dean of Science, ${ }^{13}$ Office of the President, Pennsylvania State University, University Park, PA 16802. ${ }^{4}$ Chancellor's Science Scholars Program, ${ }^{6}$ Department of Psychology and Neuroscience, ${ }^{7}$ Office of Undergraduate Education, College of Arts and Sciences, ${ }^{10}$ Office of the Chancellor, ${ }^{11}$ Department of Chemistry, ${ }^{12}$ Department of Biology (current address), University of North Carolina, Chapel Hill, Chapel Hill, NC 27559.*Email: summers@umbc.edu.
}

and backgrounds who are interested in issues of diversity and inclusion in STEM. Since inception (1989 through summer 2018), $70.8 \%$ of the 1,490 STEM undergraduates who enrolled in MYS have been URM. Most of the 879 URMs from the first 26 cohorts were retained in the program through graduation and earned science or engineering bachelor's (B.S.) degrees (739 students, 84.1\%), and most of these graduates (560 students, $75.8 \%$ ) matriculated to graduate or professional programs (47.7\% Ph.D., 13.9\% M.D.Ph.D., $19.1 \%$ masters, $19.3 \%$ medical or other professional programs). Qualified students (selection metrics included high school GPAs, SAT scores, prior research experience, expressed interest in research careers in STEM, and interviews with faculty, staff, and students, among others) who declined MYS offers and attended other universities were half as likely to graduate with a STEM degree and approximately 5 times less likely to pursue or complete STEM graduate degrees (8, 11). UMBC is the top undergraduate school of origin of African American M.D.-Ph.D. recipients in the U.S., and the second-ranked school of origin of African American STEM Ph.D. recipients (behind Howard University, an MSI) (12).

\section{PARTNERING INSTITUTIONS}

UMBC is a medium-sized High Research (Carnegie classification) university with a diverse student body $(\sim 11,000$ undergraduates: $17.2 \%$ African American, $7.1 \%$ Hispanic, $29.0 \%$ total URM; $\sim 2,500$ graduate students) and a long- standing African American president. PSU and UNC are Carnegie Very High Research universities with historically non-URM leadership. UNC has a larger but less diverse student body $(\sim 19,000$ undergraduates: 7.9\% African American, 7.2\% Hispanic, $15.6 \%$ total URM; 8,500 graduate students). From 2002-2011, UNC produced an average of 99 graduates/yr who went on to earn STEM Ph.D. degrees, of which 6/yr on average were African American (10). PSU is geographically more isolated and has a much larger and even less diverse student body ( 41,000 undergraduates: $4.7 \%$ African American, $6.8 \%$ Hispanic, $<15 \%$ total URM; $\sim 14,500$ graduate students). Although PSU was among the top five U.S. schools of origin of B.S. undergraduates who earned STEM Ph.D. degrees from 2002-2011 (averaging 193 Ph.D. degrees/yr), only 4/yr on average were African American (10). There was a prevailing sense among some leadership that the institution was too isolated, homogeneous, and under-prepared for a MYS-like program to be effective. Despite their low numbers, UNC and PSU ranked among the top 20 majority schools of origin of African American B.S. recipients who went on to earn STEM Ph.D. degrees (10).

\section{PARTNERSHIP ACTIVITIES}

Initial historical assessments of institutional STEM student demographics, academic performance, and retention at PSU and UNC identified disparities for minority students and were critical drivers for broadening support and instituting new programs: The Millennium Scholars Program (MLN) at PSU and the Chancellors Science Scholars Program (CSS) at UNC. Both were designed to replicate or closely adapt all major components of the Meyerhoff program including (i) establishment of key administrators and senior faculty as program champions, (ii) allocation of space and funding for staff, scholarships, activities, and assessment, (iii) recruitment of diverse staff who can serve as effective mentors and bridge cultural divides, (iv) targeted student recruitment and selection activities, (v) cohort building, including intensive pre-matriculation summer education and mentoring activities (Summer Bridge), (vi) early placement in research labs and summer internships, (vii) intensive academic advising and counseling, (viii) community service, and (ix) regular summative and formative program evaluations. Like MYS, MLN and CSS are strength-based programs that include students interested in URM issues in STEM regardless of gender, ethnicity, or background.

Student applicants to all three programs were selected on the basis of academic merit, STEM research and social justice interests, and pre-matriculation interviews. Some 
component details varied slightly due to differences in resources, campus cultures, and degree offerings, but in all cases the variations were designed to maintain intended component outcomes. Examples include differences among academic requirements for program admission and retention (GPA requirements ranging from 3.2 to 3.5 among programs), on-campus housing requirements (ranging from Freshman/Sophomore only to 4-years of required on-campus housing), and approaches to social justice education (a component of cohort-building and mentoring; activities included differing combinations of seminars, workshops, coursework, and/or student debates). Some components evolved over time; for example, the early MYS cohorts (including Cohorts 1-4) received full tuition/housing support, whereas more recent cohorts (including Cohorts 25-28) received tiered awards based on academic merit. MLN scholars received full tuition and housing support and CSS Scholars received partial tuition/housing support.

The partnership included several weeks of faculty and staff training at UMBC, with additional training at UNC and PSU. MLN and CSS faculty and staff were embedded in portions of the MYS Student Selection and Summer Bridge events at UMBC. Staff also met biweekly by video conference to discuss programmatic issues, evaluation team members met monthly to develop and implement evaluation plans, and faculty leadership met regularly by phone and in person to address administrative goals. Summer retreats were held involving participants from all three campuses. The Howard Hughes Medical Institute (HHMI) hosted annual staff/leadership meetings, and an external advisory board comprising experts in inclusive practices participated in local institutional events aimed at raising awareness.

\section{STUDENT OUTCOMES}

Most program students on all three campuses majored in Biology, Chemistry, Computer Science, Mathematics, Physics, Statistics, or a combination of these areas. MLN and MYS included more engineering majors than CSS $(35 \%, 27 \%$, and $4 \%$, respectively; see Supplementary Tables S1-S5), and MYS and CSS included a small number of students in other STEM-intensive majors (Psychology and Neuroscience, Geological Sciences, and Interdisciplinary STEM Studies) (14\% and $22.5 \%$, respectively).
Comparisons with Meyerhoff: Initial cohort sizes and growth closely paralleled those of MYS Cohorts 1-4, and minority participation in CSS and MLN grew from $\sim 65 \%$ (Cohort-1) to $80 \%$ (Cohort-4), which exceeds presentday MYS URM participation ( $72 \%$ for Cohorts 25-28; see Supplementary Figure S1, B) (throughout this paper, statistical significance was established by $\mathrm{t}$ tests and $\chi^{2}$ analyses, as appropriate, with $\mathrm{p}<.05$ as the threshold; see figure captions and supplementary materials for statistics and other details). STEM retention rates of the CSS and MLN cohorts were also similar to those of MYS (Supplementary Table S6, S7 and Fig. S1). Notably, the average GPAs of the first MLN and CSS cohorts significantly exceeded that of MYS, Fig. 1A (MYS vs MLN: $\mathrm{t}=3.8, \mathrm{p}<0.001 ;$ MYS vis CSS: $\mathrm{t}=4.57, \mathrm{p}<0.001$ ), and the 4-year STEM graduation rates for the first CSS and MLN cohorts $(67 \%$ and $80 \%$, respectively) also exceeded that of MYS Cohort-1 (31\%) (MYS vs MLN: $\chi^{2}=7.4, p<0.05$; MYS vs CSS: $\chi^{2}=3.9, p<0.05$ ) and were similar to present day MYS 4-year graduation rates (72\%), Fig. 1B. Importantly, the percentage of CSS Cohort-1 students who matriculated to Ph.D. and M.D.-Ph.D. programs after four years $(21 \%)$ compares favorably to that of MYS Cohort-1 (10\%) $\left(\chi^{2}=0.24, p=0.62\right)$, and MLN Cohort-1 matriculation outcomes (50\% to Ph.D. or M.D.-Ph.D. programs) greatly exceeded those of MYS Cohort-1 $\chi^{2}=5.39 \mathrm{p}<$ $0.05)$ and were similar to present-day outcomes (48\%), Fig. 1C. This reflects a key advantage of the partnership. At the time MYS was initiated, UMBC had a poor history of URM performance in STEM -- only one African American UMBC graduate had earned a STEM Ph.D. in the 25-year history of the university, and Black students held sit-ins to protest perceptions of racism. It took several years to test and implement MYS activities and to achieve broader faculty buy-in. In contrast, lessons learned at UMBC were immediately implemented at UNC and PSU, and the MYS performance history stimulated early faculty and administrative buy-in.

Comparisons with Institutionally-Matched Control Groups: To further test program efficacies, MYS, MLN and CSS student outcomes were compared with institutionally matched

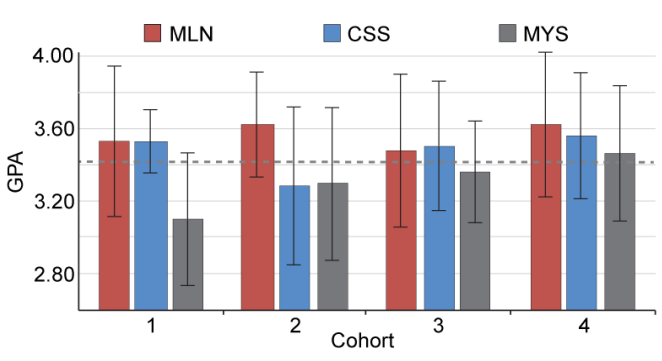

$C$

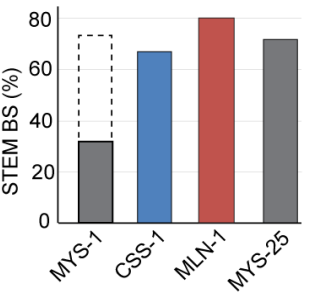

C

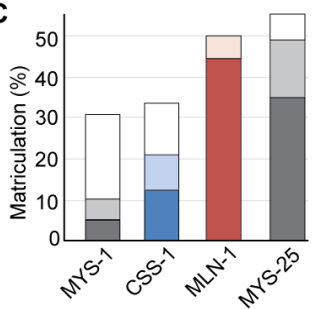

gure. 1. Comparisons with Meyerhoff. (A) Average GPAs (+/dev) of STEM-retained MLN and CSS Cohort 1 scholars exceeded e (dashed line). Subsequent cohorts of all three programs had milar GPAs. (B) STEM B.S. degrees earned after four years (shown as a percentage of the entering cohort). During initial ars of MYS development, many students required more than X). (C) Matriculation to Ph.D. (dark shades), M.D.-Ph.D. (light hades), and M.D./professional programs (white) (shown as a rcentage of the entering cohort). the basis of similar ethnicity, gender, academic interest, and entering academic credentials (high school GPA and SAT scores; see Supplementary Materials for details). In all cases, average cohort STEM retention (9194\%) and average cohort GPA for STEMretained students (3.48-3.59) were substantially greater for program participants regardless of URM or gender status (non-cohort retention $=78-80 \%$; GPA $=3.15-3.22$ ) (retention statistics: $\chi^{2}=8.7-23.3 ; \mathrm{p}<0.01$; GPA statistics: $\mathrm{t}>5.7, \mathrm{p}<0.001$; Table S8), Fig. 2. URM program participants exhibited a substantial GPA benefit $(\sim 3.45$ versus $\sim 3.05$ for matched non-cohort URM students; $\mathrm{t}>$ $4.6, \mathrm{p}<0.001$ ), and GPAs of female program participants $(\sim 3.55)$ were also considerably higher than those of female non-participants $(\sim 3.28)$ ( $\mathrm{t}>4.5, \mathrm{p}<0.001$ for all comparisons), Fig. 2.

\section{KEYS TO SUCCESS}

Partnership activities and efficacy were evaluated on the basis of confidential interviews with university administrators, faculty, and program staff (see Supplementary Materials). The following factors were considered most important for program success:

(1) Commitment to the entire MYS 
model. MYS student surveys indicate that some programmatic components are foundational for all students (e.g., Summer Bridge, community building) whereas others differentially impact students, apparently due to differences in background, culture, and preparation $(11,13)$. To ensure broadest impact, all MYS elements were replicated or closely adapted by MLN and CSS.

(2) Sufficient and sustained administrative support. MLN and CSS programs were initiated with significant institutional resources $(\$ 0.5 \mathrm{M}$ year-1 state/institutional funding) that expanded to $\$ 2.0 \mathrm{M}$ (UNC) and $\$ 2.6 \mathrm{M}$ (PSU) by Year-4, both exceeding present-day MYS state/institutional expenditures (\$1.5M) (MYS relies more heavily on grants and contracts), Fig. S2. MLN and CSS were made explicit Capital Campaign targets in 2017 , leading to endowments of $\$ 7.1 \mathrm{M}$ and $\$ 15.5 \mathrm{M}$, respectively, after only two years of fundraising. Importantly, upon upper-administrative turnover, both institutions recruited new leaders (including a new Chancellor at UNC and President at PSU) who expanded programmatic support. These activities conveyed strong campuswide messages that inclusive excellence is an institutional priority -- not only of on-campus leadership, but of the governing boards that provide institutional oversight.

(3) Recruitment of full-time program staff. Early traction was critically dependent on the ability of the Program Director to develop and maintain strong relationships with a range of constituents including administrators, potential donors, faculty, parents, students, and partnering colleagues. Staff were empowered by direct access to upper administration. Students benefited from program staff with similar experiences navigating issues of ethnicity and culture.

(4) Immersive up-front inter-institutional training and sustained guidance. Faculty and staff on both campuses indicated that training at UMBC was critical for understanding and developing MYS-like student activities and mentoring approaches. Biweekly staff meetings provided guidance and technical support and helped MLN and CCS staff respond to student and programmatic needs.

(5) Breadth of faculty participation. Faculty leadership across participating departments and colleges on both campuses played important roles in developing and championing the programs. Faculty were integrated in a wide range of programmatic activities including student recruitment, summer bridge, fundraising efforts, program administration, and workshops to raise
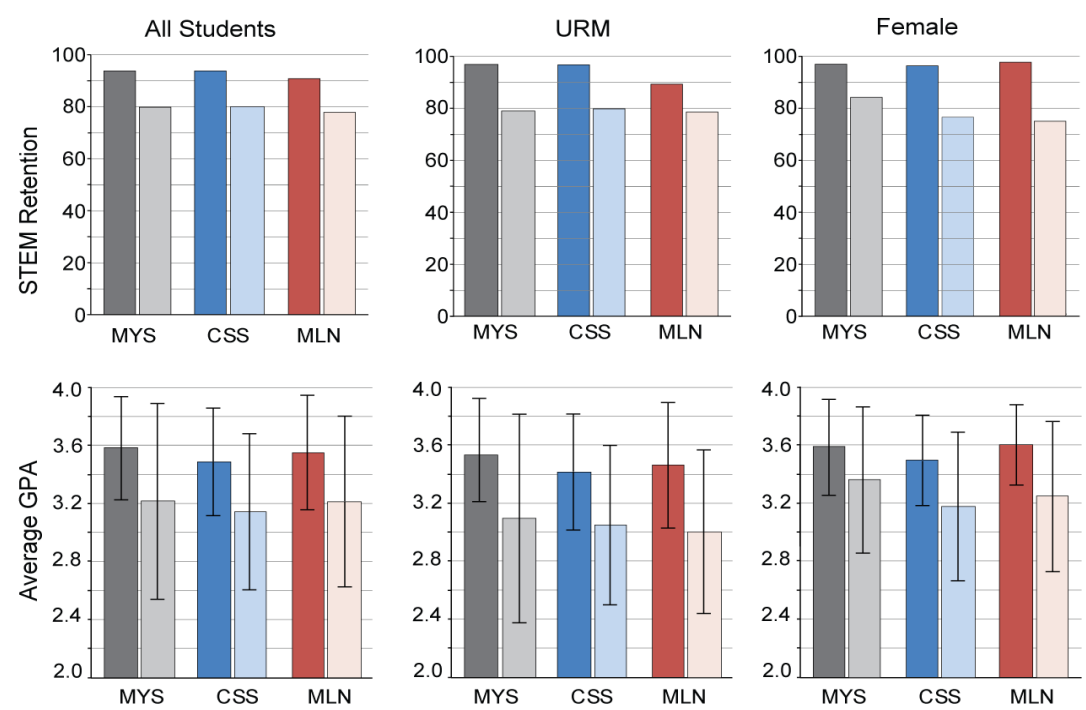

Figure 2. Impact on student outcomes. Comparisons of cohort STEM retention rates (top row) and average GPAs (bottom row, +/- stdev) of MLN and CSS Cohort 1-4 and MYS Cohort 23-26 students (dark shade) versus demographically, academically, and interest-matched institutional non-cohort control groups (light shade). Outcomes are shown for all students (left column), URMs (center), and females (right). Retention is defined as the number of STEM-retained students divided by the initial summer bridge size (entering program students) or initial non-program control group sizes.

awareness about ethnicity/gender issues in STEM. They engaged students in early (Year1) and sustained research experiences and explored pedagogical practices that appear to differentially impact URM learning and academic performance (14).

\section{CONCLUSIONS}

We have shown that MYS can be adopted at institutions that are much different from UMBC, with outcomes immediately matching or exceeding MYS. Future assessments will determine if retention and performance of non-program URMs improve at UNC and PSU as institutional climate and expectations evolve, as occurred at UMBC (7). Stimulated by these outcomes, new partnerships with the University of California Berkeley and the University of California San Diego have been initiated to assess the feasibility of long-distance inter-institutional mentoring, and mechanisms to support additional partnerships are being explored.

Strategies for improving URM persistence in different settings and among students with different levels of preparation have been described (15). Approaches that leverage lessons learned from successful programs with immersive inter-institutional partnering could serve as a general paradigm for expanding inclusive excellence in STEM.

REFERENCES AND NOTES

1. D. A. Coleman, Population and Development Review, 35, 449 (2009).
2. Does the EU Need more STEM Graduates? European Commission Report by the Danish Technological Institute (2015)

3. H. A. Valantine, F. S. Collins, Proc. Natl. Acad. Sci. U.S.A. 112,12240 (2015)

4. A. W. Woolley, C. F. Chabris, A. Pentland, N. Hashmi, T. W. Malone, Science 330, 686 (2010).

5. L. Hong, S. E. Page Proc. Natl. Acad. Sci. U.S.A. 101, 16385 (2004).

6. R. B. Freeman, W. Huang, Nature 513, 305 (2014).

7. M. F. Summers, F. A. I. Hrabowski, Science 311 1870 (2006).

8. K. I. Maton et al., CBE Life Sci Ed 15, (2016).

9. https://www2.ed.gov/about/offices/list/ocr/edlite-minorityinst.html

10. 2014 Survey of Earned Doctorates, NSF InfoBrief 13-323, National Science Foundation, Washington D.C., 2016, Tables 6,8.

11. K.I. Maton, S. A. Pollard, T. V. McDougall Weise, F. A. Hrabowski, Mt Sinai J Med 79, 610 (2012).

12. F. A. Hrabowski, III, P. H. Henderson, Issues in Sci. \& Tech. 35, 67-72 (2019)

13. K.I. Maton, M.R. Sto Domingo, K.E. Stolle-McAllister, J.L. Zimmerman, F.A. Hrabowski. J Women Minor. Sci. Eng. 15, 15 (2009).

14. S. L. Eddy, K. A. Hogan, CBE-Life Sci. Ed. 13, 453 (2014).

15. M. Estrada et al., CBE Life Sci. Ed. 15, 1-10, (2016), doi: $\underline{10.1187 / \text { cbe.16-01-0038 }}$

\section{ACKNOWLEDGMENTS}

The authors acknowledge financial and programmatic support from the Howard Hughes Medical Institute (HHMI). Program and institutional staff who assisted with data collection and program operation are acknowledged in the Supplementary Materials.

\section{SUPPLEMENTARY MATERIALS}

$x x x x x x x x x x x$

$10.1126 /$ science aar5540s 